PROCEEDINGS OF THE

AMERICAN MATHEMATICAL SOCIETY

Volume 131, Number 6, Pages 1687-1694

S 0002-9939(02)06741-2

Article electronically published on October 1, 2002

\title{
ANDRÉ-QUILLEN HOMOLOGY VIA FUNCTOR HOMOLOGY
}

\author{
TEIMURAZ PIRASHVILI
}

(Communicated by Paul Goerss)

\begin{abstract}
We obtain André-Quillen homology for commutative algebras using relative homological algebra in the category of functors on finite pointed sets.
\end{abstract}

\section{INTRODUCTION}

Let $\Gamma$ be the small category of finite pointed sets. For any $n \geq 0$, let $[n]$ be the set $\{0,1, \ldots, n\}$ with basepoint 0 . We assume that the objects of $\Gamma$ are the sets $[n]$. A left $\Gamma$-module is a covariant functor $\Gamma \rightarrow$ Vect to the category of vector spaces over a field $K$. For a left $\Gamma$-module $F$ we put

$$
\pi_{0}(F):=\operatorname{Coker}\left(d_{0}-d_{1}+d_{2}: F([2]) \rightarrow F([1])\right),
$$

where $d_{1}$ is induced by the folding map [2] $\rightarrow$ [1], 1,2 $\mapsto 1$ while $d_{0}$ and $d_{2}$ are induced by the projection maps [2] $\rightarrow$ [1] given respectively by $1 \mapsto 1,2 \mapsto 0$ and $1 \mapsto 0,2 \mapsto 1$. The category $\Gamma$ - $\bmod$ of left $\Gamma$-modules is an abelian category with enough projective and injective objects. Therefore one can form the left derived functors of the functor $\pi_{0}: \Gamma$ - $\bmod \rightarrow$ Vect, which we will denote by $\pi_{*}$. Thanks to [5] and [6] we know that $\pi_{*} F$ is isomorphic to the homotopy of the spectrum corresponding to the $\Gamma$-space $F$ according to Segal (see [9] and [1]).

Let $A$ be a commutative algebra over a ground field $K$ and let $M$ be an $A$ module. There exists a functor $\mathcal{L}(A, M): \Gamma \rightarrow$ Vect, which assigns $M \otimes A^{\otimes n}$ to $[n]$ (see [3] or section 3). Here all tensor products are taken over $K$. It was proved in [7] that $\pi_{*}(\mathcal{L}(A, M))$ is isomorphic to a brave new algebra version of AndréQuillen homology $\mathrm{H}_{*}^{\Gamma}(A, M)$ constructed by Alan Robinson and Sarah Whitehouse [10]. The main result of this paper shows that a similar isomorphism also exists for André-Quillen homology if one takes an appropriate relative derived functor of the same functor $\pi_{0}: \Gamma-\bmod \rightarrow$ Vect.

\section{A CLASS OF PROPER EXACT SEQUENCES}

Thanks to the Yoneda lemma, $\Gamma^{n}, n \geq 0$, are projective generators of the category $\Gamma$-mod. Here

$$
\Gamma^{n}:=K\left[\operatorname{Hom}_{\Gamma}([n],-)\right]
$$

Received by the editors October 13, 2001 and, in revised form, January 17, 2002

2000 Mathematics Subject Classification. Primary 13D03, 18G25.

The author was partially supported by the grant INTAS-99-00817 and by the TMR network K-theory and algebraic groups, ERB FMRX CT-97-0107. 
and $K[S]$ denotes the free vector space spanned by a set $S$. For left $\Gamma$-modules $F$ and $T$ one defines the pointwise tensor product $F \otimes T$ to be the left $\Gamma$-module given by $(F \otimes T)([n])=F([n]) \otimes T([n])$. Since $\Gamma^{n} \otimes \Gamma^{m} \cong \Gamma^{n+m}$ one sees that the tensor product of two projective left $\Gamma$-modules is still projective. We also have $\Gamma^{n} \cong\left(\Gamma^{1}\right)^{\otimes n}$.

A partition $\lambda=\left(\lambda_{1}, \cdots, \lambda_{k}\right)$ is a sequence of natural numbers $\lambda_{1} \geq \cdots \geq \lambda_{k} \geq 1$. The sum of partition is given by $s(\lambda):=\lambda_{1}+\cdots+\lambda_{k}$, while the group $\Sigma(\lambda)$ is a product of the corresponding symmetric groups

$$
\Sigma(\lambda):=\Sigma_{\lambda_{1}} \times \cdots \times \Sigma_{\lambda_{k}}
$$

which is identified with the Young subgroup of $\Sigma_{s(\lambda)}$. Let us observe that $\Sigma_{n}=$ $A u t_{\Gamma}([n])$ and therefore $\Sigma_{n}$ acts on $\Gamma^{n} \cong\left(\Gamma^{1}\right)^{\otimes n}$. For a partition $\lambda$ with $s(\lambda)=n$ we let $\Gamma(\lambda)$ be the coinvariants of $\Gamma^{n}$ under the action of $\Sigma(\lambda) \subset \Sigma_{n}$.

For a vector space $V$ we let $S^{*}(V), \Lambda^{*}(V)$ and $D^{*}(V)$ be respectively the symmetric, exterior and divided power algebra generated by $V$. Let us recall that $S^{n}(V)=\left(V^{\otimes n}\right) / \Sigma_{n}$ is the space of coinvariants of $V^{\otimes n}$ under the action of the symmetric group $\Sigma_{n}$, while $D^{n}(V)=\left(V^{\otimes n}\right)^{\Sigma_{n}}$ is the space of invariants. Moreover for a partition $\lambda=\left(\lambda_{1}, \cdots, \lambda_{k}\right)$ we put

$$
S^{\lambda}:=S^{\lambda_{1}} \otimes \cdots \otimes S^{\lambda_{k}} .
$$

We similarly define $\Lambda^{\lambda}$ and $D^{\lambda}$. It follows from the definition that

$$
\Gamma(\lambda) \cong S^{\lambda} \circ \Gamma^{1} \text {. }
$$

In particular $\Gamma(1, \cdots, 1) \cong \Gamma^{n}$ and $\Gamma(n) \cong S^{n} \circ \Gamma^{1}$.

Let

$$
0 \rightarrow T_{1} \rightarrow T \rightarrow T_{2} \rightarrow 0
$$

be an exact sequence of left $\Gamma$-modules. It is called a $\mathcal{Y}$-exact sequence if for any partition $\lambda$ with $s(\lambda)=n$ the induced map

$$
T([n])^{\Sigma(\lambda)} \rightarrow T_{2}([n])^{\Sigma(\lambda)}
$$

is surjective. Here and elsewhere, $M^{G}$ denotes the subspace of $G$-fixed elements of a $G$-module $M$. For a $\mathcal{Y}$-exact sequence $0 \rightarrow T_{1} \rightarrow T \rightarrow T_{2} \rightarrow 0$ the sequence

$$
0 \rightarrow T_{1}([n])^{\Sigma(\lambda)} \rightarrow T([n])^{\Sigma(\lambda)} \rightarrow T_{2}([n])^{\Sigma(\lambda)} \rightarrow 0
$$

is also exact. Following to Section XII.4 of 4 we introduce the relative notions. An epimorphism $f: F \rightarrow T$ is called a $\mathcal{Y}$-epimorphism if

$$
0 \rightarrow \operatorname{Ker}(f) \rightarrow F \rightarrow T \rightarrow 0
$$

is a $\mathcal{Y}$-exact sequence. Similarly, a monomorphism $f: F \rightarrow T$ is called a $\mathcal{Y}$ monomorphism if

$$
0 \rightarrow F \rightarrow T \rightarrow \operatorname{Coker}(f) \rightarrow 0
$$

is a $\mathcal{Y}$-exact sequence. A morphism $f: F \rightarrow T$ is called a $\mathcal{Y}$-morphism if $F \rightarrow \operatorname{Im}(f)$ is a $\mathcal{Y}$-epimorphism and $\operatorname{Im}(f) \rightarrow T$ is a $\mathcal{Y}$-monomorphism. A left $\Gamma$-module $Z$ is called $\mathcal{Y}$-projective if for any $\mathcal{Y}$-epimorphism $f: F \rightarrow T$ and any morphism $g: Z \rightarrow T$ there exists a morphism $h: Z \rightarrow F$ such that $g=f h$.

Lemma 2.1. $\quad$ i) If a short exact sequence is isomorphic to a $\mathcal{Y}$-exact sequence, then it is also a $\mathcal{Y}$-exact sequence.

ii) A split short exact sequence is $\mathcal{Y}$-exact.

iii) A composition of two $\mathcal{Y}$-epimorphisms is still a $\mathcal{Y}$-epimorphism. 
iv) If $f$ and $g$ are two composable epimorphisms and $f g$ is a $\mathcal{Y}$-epimorphism, then $f$ is also a $\mathcal{Y}$-epimorphism.

v) A composition of two $\mathcal{Y}$-monomorphisms is still a $\mathcal{Y}$-monomorphism.

vi) If $f$ and $g$ are two composable monomorphisms and $f g$ is a $\mathcal{Y}$-monomorphism, then $g$ is also a $\mathcal{Y}$-monomorphism.

Proof. The properties i)- iv) are clear. Let $f: B \rightarrow C$ and $g: A \rightarrow B$ be monomorphisms. One can form the following diagram:

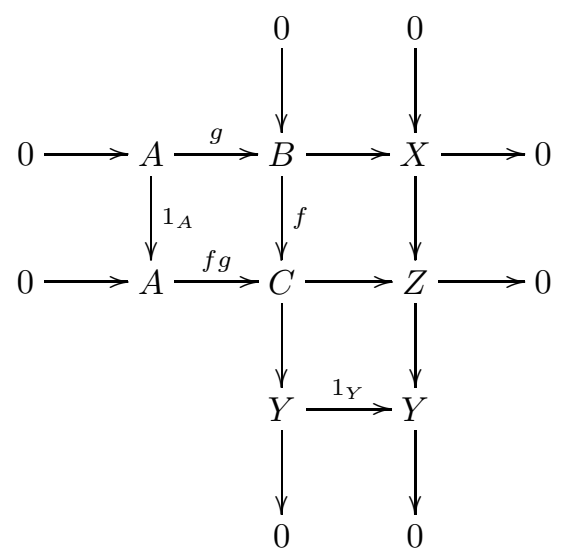

Assume $f$ and $g$ are are $\mathcal{Y}$-monomorphisms; then for any partition $\lambda$ with $s(\lambda)=n$ one has a commutative diagram:



The diagram chasing shows that $h$ is an epimorphism and therefore $f g$ is a $\mathcal{Y}$ monomorphism and $\mathrm{v}$ ) is proved. Assume now that $f g$ is a $\mathcal{Y}$-monomorphism. 
Then we have the following commutative diagram:



The diagram chasing shows that $l$ is an epimorphism and therefore $f$ is a $\mathcal{Y}$ monomorphism and therefore we get vi).

As an immediate corollary we obtain that the class of all $\mathcal{Y}$-exact sequences is proper in the sense of Mac Lane [4]. We now show that there are enough $\mathcal{Y}$ projective objects.

Lemma 2.2. $\quad$ i) For any partition $\lambda$ the left $\Gamma$-module $\Gamma(\lambda)$ is a $\mathcal{Y}$-projective object.

ii) A morphism $f: F \rightarrow T$ of left $\Gamma$-modules is a $\mathcal{Y}$-epimorphism iff for any partition $\lambda$ the induced morphism

$$
\operatorname{Hom}_{\Gamma-\bmod }(\Gamma(\lambda), F) \rightarrow \operatorname{Hom}_{\Gamma-\bmod }(\Gamma(\lambda), T)
$$

is an epimorphism.

iii) For any left $\Gamma$-module $F$ there is a $\mathcal{Y}$-projective object $Z$ and a $\mathcal{Y}$-epimorphism $f: Z \rightarrow F$.

iv) Any projective $\mathcal{Y}$-module is a direct summand of the sum of objects of the form $\Gamma(\lambda)$.

$\mathrm{v})$ The tensor product of two $\mathcal{Y}$-projective left $\Gamma$-modules is still $\mathcal{Y}$-projective.

Proof. Let $\lambda$ be a partition with $s(\lambda)=n$. By definition $\Gamma(\lambda)=H_{0}\left(\Sigma(\lambda), \Gamma^{n}\right)$. Hence for any left $\Gamma$-module $F$ one has

$$
\operatorname{Hom}_{\Gamma-\bmod }(\Gamma(\lambda), F) \cong H^{0}\left(\Sigma(\lambda), \operatorname{Hom}_{\Gamma-\bmod }\left(\Gamma^{n}, F\right) \cong F(n)^{\Sigma(\lambda)} .\right.
$$

The assertions i) and ii) are immediate consequences of this isomorphism. To prove iii) we set

$$
X(\lambda):=\operatorname{Hom}_{\Gamma-\bmod }(\Gamma(\lambda), F) .
$$

Moreover, for each $x \in X(\lambda)$ we let $f_{x}: \Gamma(\lambda) \rightarrow F$ be the corresponding morphism. Take $Z=\bigoplus_{\lambda} \bigoplus_{x \in X(\lambda)} \Gamma(\lambda)$. Then the collection $f_{x}, x \in X(\lambda)$, yields the morphism $f: Z \rightarrow F$. We have to show that it is a $\mathcal{Y}$-epimorphism. Let $g: \Gamma(\lambda) \rightarrow F$ be a morphism of left $\Gamma$-modules. By ii) we need to lift $g$ to $Z$. By our construction $g \in X(\lambda)$ and therefore the inclusion $\Gamma(\lambda) \rightarrow Z$ corresponding to the summand $g \in X(\lambda)$ is an expected lifting and iii) is proved. The proof of iii) shows that one can assume $P$ to be a sum of $\Gamma^{\lambda}$ and iv) follows. To prove the last statement one observes that, for any partitions $\lambda$ and $\mu$, one has

$$
\Gamma(\lambda) \otimes \Gamma(\mu) \cong\left(\Gamma^{s(\lambda)} \otimes \Gamma^{s(\mu)}\right)^{\Sigma(\lambda) \times \Sigma(\mu)}=\left(\Gamma^{s(\lambda)+s(\mu)}\right)^{\Sigma(\lambda) \times \Sigma(\mu)}
$$

and therefore $\Gamma(\lambda) \otimes \Gamma(\mu)$ is $\mathcal{Y}$-projective. 


\section{Definition of André-Quillen homology and the functor $\mathcal{L}$}

The definition of André-Quillen homology is based on the framework of homotopical algebra [8] and it is given as follows. We let $C_{*}\left(V_{*}\right)$ be the chain complex associated to a simplicial vector space $V_{*}$. Let $A$ be a commutative algebra over a ground field $K$ and let $M$ be an $A$-module. A simplicial resolution of $A$ is an augmented simplicial object $P_{*} \rightarrow A$ in the category of commutative algebras, which is a weak equivalence (in other words $C_{*}\left(P_{*}\right) \rightarrow A$ is a weak equivalence). A simplicial resolution is called free if $P_{n}$ is a polynomial algebra over $K$ for all $n \geq 0$. Any commutative algebra possesses a free simplicial resolution which is unique up to homotopy. Then the André-Quillen homology is defined by

$$
\mathrm{D}_{*}(A, M):=H_{*}\left(C_{*}\left(\Omega_{P_{*}}^{1} \otimes_{P_{*}} M\right)\right),
$$

where $\Omega^{1}$ is the Kähler 1-differential and $P_{*} \rightarrow A$ is a free simplicial resolution. In dimension 0 we have $\mathrm{D}_{0}(A, M) \cong \Omega_{A}^{1} \otimes_{A} M$.

As we mentioned above the functor $\mathcal{L}(A, M): \Gamma \rightarrow$ Vect is given on objects by $[n] \mapsto M \otimes A^{\otimes n}$. For a pointed map $f:[n] \rightarrow[m]$, the action of $f$ on $\mathcal{L}(A, M)$ is given by

$$
f_{*}\left(a_{0} \otimes \cdots \otimes a_{n}\right):=b_{0} \otimes \cdots \otimes b_{m},
$$

where $b_{j}=\prod_{f(i)=j} a_{i}, j=0, \cdots, n$.

Example 3.1. Let $M=A=K[t]$. In this case one has an isomorphism

$$
\mathcal{L}(K[t], K[t]) \cong S^{*} \circ \Gamma^{1} .
$$

To see this isomorphism, one observes that $\Gamma^{1}$ assigns the free vector space on a set $[n]$ to $[n]$ and therefore both functors in question assign the ring $K\left[t_{0}, \cdots, t_{n}\right]$ to $[n]$. An important consequence of this isomorphism is the fact that the functor $\mathcal{L}(K[t], K[t])$ is $\mathcal{Y}$-projective.

Lemma 3.2. For any commutative algebra $A$ and any $A$-module $M$, one has a natural isomorphism $\pi_{0}(\mathcal{L}(A, M)) \cong \Omega_{A}^{1} \otimes_{A} M$.

Proof. By the definition we have $\pi_{0}(\mathcal{L}(A, M))=\operatorname{Coker}\left(b: M \otimes A^{\otimes 2} \rightarrow M \otimes A\right)$, where $b(m \otimes a \otimes b)=m a \otimes b-m \otimes a b+m b \otimes a$. Since

$$
a d b \otimes m \mapsto(\operatorname{ma} \otimes b) \bmod \operatorname{Im}(b)
$$

yields the isomorphism $\Omega_{A}^{1} \otimes_{A} M \rightarrow \operatorname{Coker}(b)$, the result follows.

Lemma 3.3. $\quad$ i) Let $A$ be a commutative algebra and let

$$
0 \rightarrow M_{1} \rightarrow M \rightarrow M_{2} \rightarrow 0
$$

be a short exact sequence of A-modules. Then

$$
0 \rightarrow \mathcal{L}\left(A, M_{1}\right) \rightarrow \mathcal{L}(A, M) \rightarrow \mathcal{L}\left(A, M_{2}\right) \rightarrow 0
$$

is a $\mathcal{Y}$-exact sequence.

ii) Let $f: B \rightarrow A$ be a surjective homomorphism of commutative algebras. Then for any A-module $M$ the induced morphism of left $\Gamma$-modules

$$
\mathcal{L}(B, M) \rightarrow \mathcal{L}(A, M)
$$

is a $\mathcal{Y}$-epimorphism. 
Proof. One observes that for any partition $\lambda$ with $s(\lambda)=n$ one has

$$
(\mathcal{L}(A, M)([n]))^{\Sigma(\lambda)}=\left(M \otimes A^{\otimes n}\right)^{\Sigma(\lambda)} \cong M \otimes D^{\lambda}(A) .
$$

Since we are over a field the tensor product is exact and we obtain i). By the same reason $f$ has a linear section, which also yields a linear section of $D^{\lambda}(B) \rightarrow D^{\lambda}(A)$, because $D^{\lambda}$ is a functor defined on the category of vector spaces.

\section{Relative DeRIVED FUnCTORS}

By Lemma 2.2 the class of $\mathcal{Y}$-exact sequences has enough projective objects. Thanks to 4 this allows us to construct the relative derived functors. Let us recall that an augmented chain complex $X_{*} \rightarrow F$ is called a $\mathcal{Y}$-resolution of $F$ if it is exact (that is, $H_{i}\left(X_{*}\right)=0$ for $i>0$ and $H_{0}\left(X_{*}\right) \cong F$ ) and all boundary maps $X_{n+1} \rightarrow X_{n}$ are $\mathcal{Y}$-morphisms, $n \geq 0$. It follows from Lemma 2.2 that $X_{*} \rightarrow F$ is a $\mathcal{Y}$-resolution iff for any partition $\lambda$ the augmented complex

$$
\operatorname{Hom}_{\Gamma-\bmod }\left(\Gamma(\lambda), X_{*}\right) \rightarrow \operatorname{Hom}_{\Gamma-\bmod }(\Gamma(\lambda), F)
$$

is exact. A $\mathcal{Y}$-resolution $Z_{*} \rightarrow F$ is called a $\mathcal{Y}$-projective resolution if for all $n \geq 0$ the left $\Gamma$-module $Z_{n}$ is a $\mathcal{Y}$-projective object. We define $\pi_{*}^{\mathcal{Y}}(F)$ using relative derived functors of the functor $\pi_{0}: \Gamma-\bmod \rightarrow$ Vect. In other words we put

$$
\pi_{n}^{\mathcal{Y}}(F):=H_{n}\left(\pi_{0}\left(Z_{*}\right)\right), n \geq 0,
$$

where $Z_{*} \rightarrow F$ is a $\mathcal{Y}$-projective resolution. By 4 ] this gives the well-defined functors $\pi_{n}^{\mathcal{Y}}: \Gamma-\bmod \rightarrow$ Vect, $n \geq 0$.

Lemma 4.1. If $K$ is a field of characteristic zero, then $\pi_{*}(F) \cong \pi_{*}^{\mathcal{Y}}(F)$.

Proof. In this case all exact sequences are $\mathcal{Y}$-exact, because for any finite group $G$, the functor $M \mapsto M^{G}$ is exact.

Lemma 4.2. For left $\Gamma$-modules $F, T$ one has an isomorphism

$$
\pi_{*}^{\mathcal{Y}}(F \otimes T) \cong \pi_{*}^{\mathcal{Y}}(F) \otimes T([0]) \oplus F([0]) \otimes \pi_{*}^{Y}(T) .
$$

Proof. The result in dimension 0 is known (see Lemma 4.2 of [5]). Let $Z_{*} \rightarrow F$ and $R_{*} \rightarrow T$ be $\mathcal{Y}$-projective resolutions. By Lemma $2.2 Z_{*} \otimes R_{*} \rightarrow F \otimes T$ is also a $\mathcal{Y}$-projective resolution. Thus

$$
\begin{gathered}
\pi_{*}^{\mathcal{Y}}(F \otimes T)=H_{*}\left(\pi_{0}\left(Z_{*} \otimes R_{*}\right)\right) \\
\cong H_{*}\left(\pi_{0}^{\mathcal{Y}}\left(Z_{*}\right) \otimes R_{*}([0]) \oplus Z_{*}([0]) \otimes \pi_{0}^{Y}\left(R_{*}\right)\right) \\
\cong \pi_{*}^{\mathcal{Y}}(F) \otimes T([0]) \oplus F([0]) \otimes \pi_{*}^{Y}(T),
\end{gathered}
$$

where the last isomorphism follows from the Eilenberg-Zilber theorem and Künneth theorem.

Lemma 4.3. Let $\epsilon: X_{*} \rightarrow A$ be a simplicial resolution in the category of commutative algebras and let $M$ be an A-module. Then the associated chain complex of the simplicial $\Gamma$-module $C_{*}\left(\mathcal{L}\left(X_{*}, M\right)\right) \rightarrow \mathcal{L}(A, M)$ is a $\mathcal{Y}$-resolution.

Proof. Since $\epsilon$ is a weak equivalence of simplicial algebras it is a homotopy equivalence in the category of simplicial vector spaces. Thus $M \otimes D^{\lambda}\left(X_{*}\right) \rightarrow M \otimes D^{\lambda}\left(A_{*}\right)$ is also a homotopy equivalence, for any partition $\lambda$. It follows that

$$
\mathcal{L}\left(X_{*}, M\right)([n])^{\Sigma(\lambda)} \rightarrow \mathcal{L}(A, M)([n])^{\Sigma(\lambda)}
$$

is also a homotopy equivalence of simplicial vector spaces. 
The following is our main result.

Theorem 4.4. For any commutative ring $A$ and any $A$-module $M$, there is a canonical isomorphism

$$
\mathrm{D}_{i}(A, M) \cong \pi_{i}^{\mathcal{Y}}(\mathcal{L}(A, M)), i \geq 0
$$

between the André-Quillen homology and relative derived functors of $\pi_{0}$ applied on the functor $\mathcal{L}(A, M)$.

Proof. Thanks to Lemma 3.2 the result is true for $i=0$. First consider the case when $M=A=K[t]$. In this case the André-Quillen homology vanishes in positive dimensions by definition. On the other hand $\mathcal{L}(K[t], K[t])$ is $\mathcal{Y}$-projective thanks to Example 3.1 and therefore $\pi_{i}^{\mathcal{Y}}(\mathcal{L}(A, M))$ vanishes for all $i>0$. One can use Lemma 4.2 to conclude that $\pi_{i}^{\mathcal{Y}}(\mathcal{L}(A, A))$ vanishes for all $i>0$ provided $A$ is a polynomial algebra. For the next step, we prove that the result is true if $A$ is a polynomial algebra and $M$ is any $A$-module. We have to prove that $\pi_{i}^{\mathcal{Y}}(\mathcal{L}(A, M))$ also vanishes for $i>0$. We already proved this fact if $M=A$. By additivity the functor $\pi_{i}^{\mathcal{Y}}(\mathcal{L}(A,-))$ vanishes on free $A$-modules. By Lemma 3.3 the functor $\pi_{*}^{\mathcal{Y}}(\mathcal{L}(A,-))$ assigns the long exact sequence to a short exact sequence of $A$-modules. Therefore we can consider such an exact sequence associated to a short exact sequence of $A$-modules

$$
0 \rightarrow N \rightarrow F \rightarrow M \rightarrow 0
$$

with free $F$. Since the result is true if $i=0$, one obtains by induction on $i$ that $\pi_{i}^{\mathcal{Y}}(\mathcal{L}(A, M))=0$ provided $i>0$. Now consider the general case. Let $P_{*} \rightarrow A$ be a free simplicial resolution in the category of commutative algebras. Then we have

$$
\Omega_{P_{*}}^{1} \otimes_{P_{*}} M \cong \pi_{0}^{\mathcal{Y}}\left(\mathcal{L}\left(P_{*}, M\right)\right) .
$$

Thanks to Lemma 4.3 $C_{*}\left(\mathcal{L}\left(P_{*}, M\right)\right) \rightarrow \mathcal{L}(A, M)$ is a $\mathcal{Y}$-resolution consisting of $\pi_{*}^{\mathcal{Y}}$-acyclic objects and the result follows.

The main theorem together with the main result of [7] yields:

Corollary 4.5. If Char $(K)=0$, then for any commutative algebra $A$ and any A-module $M$ one has a natural isomorphism

$$
\mathrm{D}_{*}(A, M) \cong \mathrm{H}_{*}^{\Gamma}(A, M) .
$$

This fact was also proved in [10] based on the combinatorical and homotopical analysis of the space of fully grown trees.

Remarks. i) We let $t: \Gamma^{o p} \rightarrow$ Vect be the functor which assigns the vector space of all maps $f:[n] \rightarrow K, f(0)=0$ to $[n]$. Then $t \otimes_{\Gamma} F \cong \pi_{0}(F)$ (see Proposition 2.2 of [5]). Hence $\pi_{*}^{\mathcal{Y}}$ can also be defined as the relative derived functors of the functor $t \otimes_{\Gamma}(-): \Gamma-\bmod \rightarrow$ Vect. More generally one can take any functor $T: \Gamma^{o p} \rightarrow$ Vect and define $\operatorname{Tor}_{*}^{\mathcal{Y}}(T, F)$ as the value of the relative derived functors (with respect to $\mathcal{Y}$-exact sequences) of the functor $T \otimes_{\Gamma}(-): \Gamma$ - $\bmod \rightarrow$ Vect. Then our result claims that

$$
\mathrm{D}_{*}(A, M) \cong \operatorname{Tor}_{*}^{\mathcal{Y}}(t, \mathcal{L}(A, M)) .
$$

Based on Proposition 1.15 of [5] the argument given above shows that

$$
\mathrm{D}_{*}^{\{n\}}(A, M) \cong \operatorname{Tor}_{*}^{\mathcal{Y}}\left(\Lambda^{n} \circ t, \mathcal{L}(A, M)\right),
$$


where $\mathrm{D}_{*}^{\{n\}}(A, M)$ are defined using Kähler $n$-differentials:

$$
\mathrm{D}_{*}^{\{n\}}(A, M):=H_{*}\left(C_{*}\left(\Omega_{P_{*}}^{n} \otimes_{P_{*}} M\right)\right)
$$

and for $n=1$ one recovers the main theorem.

ii) All results remains true if $K$ is any commutative ring and $A$ and $M$ are projective as $K$-modules.

\section{ACKNOWLEDGMENTS}

This work was written during my visit to the Max-Planck-Institut für Mathematik at Bonn.

\section{REFERENCES}

[1] A. K. Bousfield and E.M. Friedlander. Homotopy theory of $\Gamma$-spaces, spectra, and bisimplicial sets. Geometric applications of homotopy theory (Proc. Conf., Evanston, Ill., 1977), II, pp. 80-130, Lecture Notes in Math., 658, Springer, 1978. MR 80e:55021

[2] A. Dold and D. Puppe. Homologie nicht-additiver Funktoren, Anwendungen. Ann. Inst. Fourier 11 (1961), 201-312. MR 27:186

[3] J. - L. LodAY. Opérations sur l'homologie cyclique des algèbres commutatives. Invent. Math. 96 (1989), 205-230. MR 89m:18017

[4] S. Mac Lane. Homology. Classics in Mathematics. Springer-Verlag, Berlin, 1995. x+422 pp. MR 96d:18001

[5] T. Pirashvili. Hodge decomposition of higher order Hochschild homology. Ann. Sci. École Norm. Sup. (4) 33 (2000), no. 2, 151-179. MR 2001e:19006

[6] T. Pirashvili. Dold-Kan type theorem for $\Gamma$-groups. Math. Ann. 318 (2000), no. 2, 277-298. MR 2001i:20112

[7] T. Pirashvili and B. Richter. Robinson-Whitehouse complex and stable homotopy. Topology 39 (2000), no. 3, 525-530. MR 2001e:13019

[8] D.G. Quillen. On the (co)homology of commutative rings. AMS Proc. Sym. Pure Math. 17 (1970), 65-87. MR 41:1722

[9] G. Segal. Categories and cohomology theories. Topology 13 (1974), 293-312. MR 50:5782

[10] S. A. Whitehouse. Gamma (co)homology of commutative algebras and some related representations of the symmetric group. Thesis. University of Warwick. 1994.

Razmadze Mathematical Institute, Rukhadze Str. 1, Tbilisi 380093, Republic of GeorGIA

E-mail address: pira@rmi.acnet.ge 